\title{
Correction to: Robust control of reaction wheel bicycle robot via adaptive integral terminal sliding mode
}

\author{
Long Chen · Jun Liu - Hai Wang (1) Youhao Hu (1) - Xuefeng Zheng • \\ Mao Ye $\cdot$ Jie Zhang
}

Published online: 5 May 2021

(C) Springer Nature B.V. 2021

\section{Correction to: Nonlinear Dyn}

https://doi.org/10.1007/s11071-021-06380-9

Funding information and grant number were omitted from the original article. The original article has been corrected.

The original article can be found online at https:// doi.org/10.1007/s11071-021-06380-9.

L. Chen $\cdot$ J. Liu $\cdot$ X. Zheng

School of Electronics and Information, Hangzhou Dianzi

University, Hangzhou 310012, China

H. Wang $(\bowtie) \cdot$ Y. Hu $\cdot$ M. Ye $\cdot$ J. Zhang

Discipline of Engineering and Energy, Centre for Water-

Energy-Waste, Murdoch University, Perth,

WA 6150, Australia

e-mail: hai.wang@murdoch.edu.au 